\title{
Estrutura e persistência temporal da comunidade de peixes de três riachos do Alto Rio Tocantins, GO
}

\author{
Jean Carlos Miranda ${ }^{1,2}$ \& Rosana Mazzoni ${ }^{2,3}$ \\ ${ }^{1}$ Programa de Pós-Graduação em Biologia - Universidade do Estado do Rio de Janeiro - UERJ, \\ ${ }^{2}$ Laboratório de Ecologia de Peixes, Departamento de Ecologia, \\ Universidade do Estado do Rio de Janeiro - UERJ, \\ Av. São Francisco Xavier, 524, CEP 20550-011, Maracanã, RJ, Brasil \\ ${ }^{3}$ Autor para correspondência: Rosana Mazzoni, e-mail: mazzoni@uerj.br
}

\begin{abstract}
MIRANDA, J.C. \& MAZZONI, R. Structure and temporal persistence of a fish community in three streams from the Upper Tocantins River, GO. Biota Neotrop., 9(4): http://www.biotaneotropica.org.br/v9n4/en/ abstract?article+bn02209042009.
\end{abstract}

\begin{abstract}
Three streams from the upper Tocantins basin were studied in order to describe community structure and temporal persistence of fish species composition. Fish densities of each stream were compared between them and with other streams from Neotropical, Austral and Holartic regions. Forty six species were registered but only 18 co-occurred in the three studied streams. Two streams presented similar species composition, but temporal variations of species densities differed among them. High persistence was registered to Água Boa and Cavalo streams whereas low persistence was registered to Acaba Saco stream. No significant differences were registered for species densities of each studied stream, nonetheless, Cavalo were that tending to support the highest fish densities. The values of fish density found in the present study were very similar to those found in other geographic regions such as Temperate and Tropical areas.
\end{abstract}

Keywords: Araguaia-Tocantins basin, stream-dwelling fishes, density, stability.

MIRANDA, J.C. \& MAZZONI, R. Estrutura e persistência temporal da comunidade de peixes de três riachos do Alto Rio Tocantins, GO. Biota Neotrop., 9(4): http://www.biotaneotropica.org.br/v9n4/pt/ abstract?article+bn02209042009.

Resumo: Três riachos do alto rio Tocantins foram estudados para determinação da estrutura e persistência temporal das comunidades de peixes. As densidades das populações que compõem cada comunidade foram comparadas entre os três riachos estudados e entre os mesmos e alguns registros disponíveis para outros riachos das regiões Neotropical, Austral e Holártica. Registramos 46 espécies, sendo que apenas 18 espécies foram compartilhadas pelos três riachos. A análise de similaridade revelou que dois riachos têm composição de espécies muito similar, porém com variações temporais distintas. Os córregos Água Boa e Cavalo apresentaram alta persistência na estrutura de suas comunidades enquanto que para o córrego Acaba Saco registramos baixa persistência. Não houve diferença significativa nas densidades médias das espécies dos três córregos, sendo que o Cavalo foi aquele que apresentou tendência a suportar maiores densidades. Nossos resultados indicam que os valores de densidade registrados para os córregos estudados são similares a resultados registrados em outras regiões geográficas, como áreas Temperadas e Tropicais.

Palavras-chave: bacia Araguaia-Tocantins, peixes de riacho, densidade, persistência, estabilidade. 


\section{Introdução}

As comunidades de peixes têm sido interpretadas de acordo com a composição e/ou diversidade de espécies, bem como através dos padrões de distribuição que refletem a disponibilidade de recursos ambientais (Bayley \& Li 1992) e/ou as interações biológicas entre as espécies (Matthews 1998). Atualmente, dispomos de diversas abordagens dessa natureza para riachos da região tropical (i.e. Aranha \& Caramaschi 1997, Castro \& Casati 1997, Barretto \& Uieda 1998, Mazzoni \& Lobón-Cerviá 2000, Casatti et al. 2001, Fogaça et al. 2003, Barreto \& Aranha 2005, Braga \& Andrade 2005, Mazzoni et al. 2006, Apone et al. 2008, Rocha et al. 2009), porém, a maioria dos modelos que abordam a questão da estrutura e dinâmica das comunidades de rios ainda está amplamente baseada em padrões observados para ambientes de regiões de clima temperado e, mesmo com o aumento do número de trabalhos em regiões tropicais, não houve, até o momento, mudança nos paradigmas sobre o tema. Vannote et al. (1980) propõem o Conceito do Rio Contínuo como forma de explicar a estruturação das comunidades aquáticas em rios. Segundo esse modelo a distribuição e abundância dos organismos são diretamente influenciadas pela disponibilidade de alimento, que por sua vez é conseqüência da dinâmica do curso d'água. Esse conceito, no entanto, não leva em consideração fatos como a qualidade química da água e as interações bióticas típicas dos ambientes aquáticos (Angermeier \& Karr 1984).

Grande parte dos rios de regiões tropicais tem seus regimes hídricos regidos por precipitações sazonais e as respostas às chuvas locais têm relação direta com as peculiaridades do sistema (Payne 1986). Neste contexto ressaltam-se dois tipos de ambientes: 1) os rios de grande porte que apresentam uma dinâmica hídrica menos susceptível às precipitações locais, tendo em seus afluentes a possibilidade de abrigo compatível com as necessidades das espécies, e 2) os rios de pequeno porte que são marcadamente atingidos pelas chuvas, que muitas vezes parecem atuar como desestruturadoras do curso d'água e de sua biocenose. A diversidade de sistemas hídricos que ocorre nas regiões tropicais proporciona, dessa forma, um campo ilimitado para a formulação de hipóteses testáveis quanto às estratégias de colonização, manutenção e reposição de populações e comunidades. No médio prazo, a reunião dessa informação poderá compor um importante arcabouço teórico sobre os padrões e processos que ocorrem em sistemas tropicais.

Neste trabalho tivemos como objetivo determinar a persistência temporal da estrutura da comunidade de peixes que ocorrem em três riachos do alto Rio Tocantins e comparar nossos resultados com algumas informações sobre a ictiofauna de riachos das regiões Neotropical, Austral e Holártica.

\section{Material e Métodos}

\section{1. Área de estudo}

O Rio Tocantins tem sua nascente na serra Paranã, numa altitude de aproximadamente $1.100 \mathrm{~m}$, a cerca de $60 \mathrm{~km}$ ao norte de Brasília, e percorre cerca de $2.400 \mathrm{~km}$ desde a nascente até sua foz em Belém do Pará. A divisão de trechos para o Rio Tocantins realizada por Innocêncio (1977) considera que o alto Rio Tocantins percorre cerca de $1.060 \mathrm{~km}$, das nascentes até a cachoeira Lageado $\left(10^{\circ} \mathrm{S}\right.$ e $\left.52^{\circ} \mathrm{O}\right)$. $\mathrm{O}$ regime hidrológico da bacia é bem definido, com período de águas altas de outubro a abril e período seco de maio a setembro. A vazão média do rio Tocantins, registrada na confluência com o rio Araguaia, varia nas épocas extremas de $7860 \mathrm{~m}^{3} / \mathrm{s}$ na cheia a $784 \mathrm{~m}^{3} / \mathrm{s}$ na vazante (Innocêncio 1977).
$\mathrm{O}$ alto Rio Tocantins, na região de Serra da Mesa

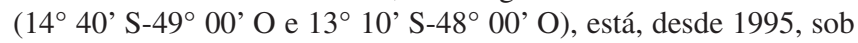
influência de um represamento para a produção de energia elétrica. Os riachos estudados no presente trabalho (córregos Acaba Saco, Cavalo e Água Boa) tiveram a composição e diversidade de espécies registradas por Miranda \& Mazzoni (2003) e encontram-se situados nas áreas adjacentes ao reservatório recém formado. A despeito do represamento ocorrido na região, o regime pluvial da área de estudo não sofreu alterações e, durante o período de estudo, manteve-se conforme a previsão e tendência histórica da região, apresentando estações chuvosa e seca bem definidas (Figura 1). A descrição detalhada das localidades de estudo bem como a composição de espécies dos riachos estudados está registrada em Miranda \& Mazzoni (2003).

\section{Programa de amostragem}

As coletas foram realizadas bimestralmente, por pesca elétrica (CA - 1.600 W, 220 V, 2-4 A) conforme descrito em Mazzoni et al. (2000), no período de dezembro de 1995 a dezembro de 1999. O período de amostragem reuniu quatro anos de estudo com a caracterização de quatro estações secas e quatro estações chuvosas. A cada ocasião amostral eram realizados mapas batimétricos, baseados em transectos transversais registrados em intervalos de $5 \mathrm{~m}$ desde a parte inferior até a parte superior do trecho amostrado, a fim de se obter dados para o cálculo da área amostrada em cada riacho estudado. A extensão dos trechos amostrados foi: córrego Acaba Saco - 105 m, córrego Cavalo - 150 m e Água Boa - 125 m. As amostragens seguiram o método das remoções sucessivas (Zippin 1958): os exemplares

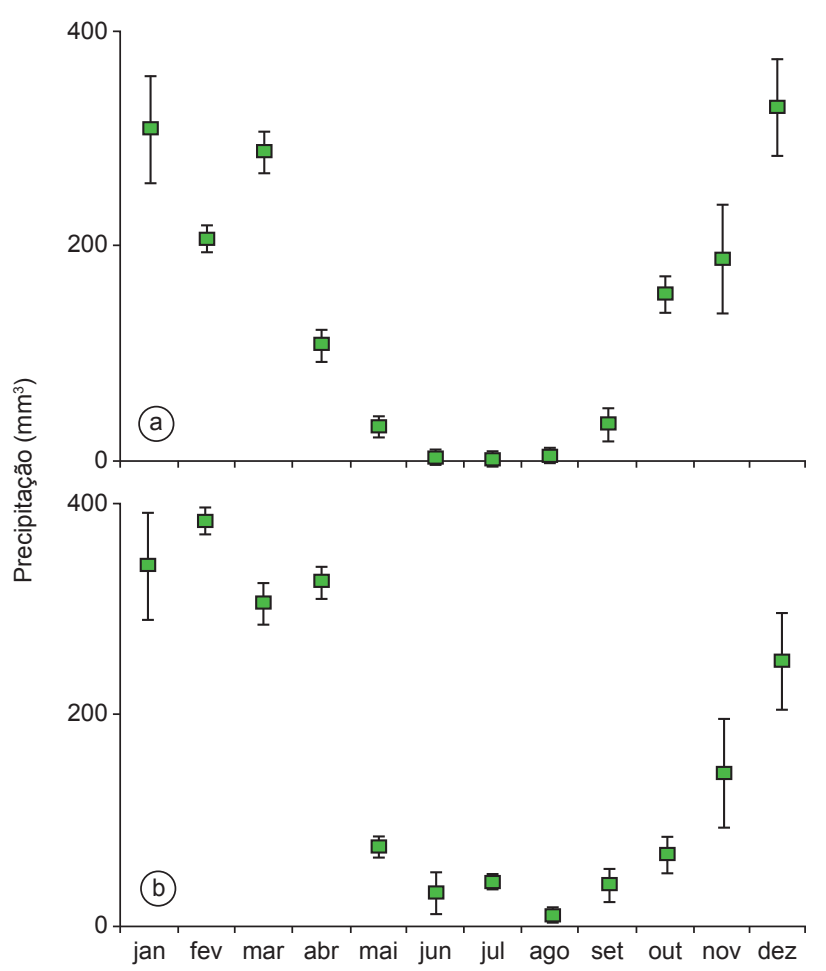

Figura 1. Valores médios da precipitação pluviométrica $\left(\mathrm{mm}^{3}\right)$ na região do alto rio Tocantins. Série histórica para a) o período 1990 a 1995 e b) período de estudo entre 1995 e 1999.

Figure 1. Mean values of rainfall $\left(\mathrm{mm}^{3}\right)$ in the upper Tocantins region. Historical series for the period a) between 1990 and 1995 and b) study period between 1995 and 1999. 
coletados eram identificados, contados e medidos considerando-se cada remoção separadamente; ao final desse procedimento todos os exemplares eram devolvidos vivos ao ambiente.

\section{Tratamento dos dados}

As abundâncias de cada população foram estimadas pelo método de Zippin (Zippin 1958), com base nas capturas sucessivas realizadas através da pesca elétrica. Essas estimativas foram realizadas segundo o modelo apresentado a seguir e só pode ser resolvido de forma iterativa:

$$
\mathrm{f}(\mathrm{x})=\left(\mathrm{N} ! /(\mathrm{N}-\mathrm{Ct}) ! * \sum_{\mathrm{i}=1}^{\mathrm{s}} \mathrm{Ci}\right) * \mathrm{p}^{\mathrm{Ct}} * \mathrm{q}^{\mathrm{s} * \mathrm{~N}} \sum_{\mathrm{i}=1}^{\mathrm{s}+1} * \mathrm{Ki}
$$

as soluções de $\mathrm{N}$ e p:

$$
\mathrm{N}=\mathrm{Ct} /\left(1-\mathrm{q}^{\mathrm{s}}\right) \mathrm{e} \mathrm{q} / \mathrm{p}-\mathrm{s}^{*} \mathrm{q}^{\mathrm{s}} /\left(1-\mathrm{q}^{\mathrm{s}}\right)=\sum_{\mathrm{i}=1}^{\mathrm{s}}(\mathrm{i}-1) * \mathrm{Ci} / \mathrm{Ct}=\mathrm{R}
$$

onde: $\mathrm{N}=$ número de indivíduos estimados, $\mathrm{Ct}=$ número de indivíduos capturados, $\mathrm{Ci}$ = número de indivíduos capturados na ocasião $\mathrm{i}, \mathrm{p}=$ probabilidade de que um indivíduo esteja na unidade amostral, $\mathrm{q}=$ complemento de $\mathrm{p}$ e $\mathrm{K}$ = capturabilidade.

Para a aplicação do método de Zippin três premissas são assumidas: i) a população amostrada é fechada, i.e. nenhum indivíduo entra ou sai do trecho amostral durante o experimento; ii) o esforço de pesca é constante, i.e. o tempo de pesca é o mesmo para todas as ocasiões; iii) a capturabilidade é constante, i.e. a probabilidade de ser capturado é igual para todos os indivíduos da população. A constância das capturabilidades foi testada através dos valores de $\mathrm{T}_{1}$ (Seber 1982), que assume uma distribuição binomial, considerando-se três ocasiões consecutivas de pesca e esforço constante. As estimativas que não apresentam $\mathrm{T}_{1}$ significativo em $\mathrm{P}<0,05$ são consideradas como estimativas não significativas e, portanto, apresentam variâncias muito elevadas. A condição de falha $(\mathrm{CF})$ atende ao modelo
[ $\Sigma(\mathrm{s}+1-2 \mathrm{i}) \mathrm{Ci}]<0$ (equação 03 ), onde $\mathrm{s}=$ número de ocasiões de pesca, $\mathrm{i}=$ ocasião de pesca (neste caso 1, 2 ou 3) e $\mathrm{Ci}=$ número de exemplares capturados na ocasião de pesca i. Esse modelo estabelece uma ponderação. O número de indivíduos capturados deve decrescer a cada ocasião, caso contrário verifica-se a $\mathrm{CF}$ e, portanto, não procede estimar a abundância da população em questão. As equações de 01 a 03 foram desenvolvidas com auxílio de um programa BASIC, para microcomputadores, apresentado em Lobón-Cerviá (1991). Os dados de abundância de cada população foram transformados em densidade (número de indivíduos $/ \mathrm{m}^{2}$ ) a partir dos valores de área calculados com base nos dados batimétricos obtidos em cada riacho e ocasião de coleta.

A similaridade na estrutura das comunidades foi analisada para as matrizes de densidade das espécies de cada riacho através do método UPGMA e distâncias de Bray Curtis (McCune \& Mefford 1997). Os dados de densidade das espécies foram analisados em escala temporal de maneira a se avaliar a persistência na estrutura das comunidades dos diferentes riachos estudados. Dessa forma os padrões de covariação interespecífica, entre os diferentes períodos amostrais, foram testados através de correlações não paramétricas de Spearman (Zar 1996).

\section{Resultados}

A lista atualizada das espécies registradas nos três riachos estudados está apresentada na Tabela 1. Dessa lista, várias espécies ainda encontram-se em poder de especialistas para descrição, pois foram identificadas como espécies novas, outras já estão com nova nomenclatura, pois foram descritas como espécies novas no âmbito do projeto que gerou essa informação e algumas ainda estão em fase de identificação. Dado o elevado número de identificações taxonômicas em andamento, para todas as espécies e/ou morfotipos aqui

Distância de Bray-Curtis
2,3E-02
$7 \mathrm{E}-01$
$1,4 \mathrm{E}+00$
$2 E+00$
$2,7 \mathrm{E}+00$

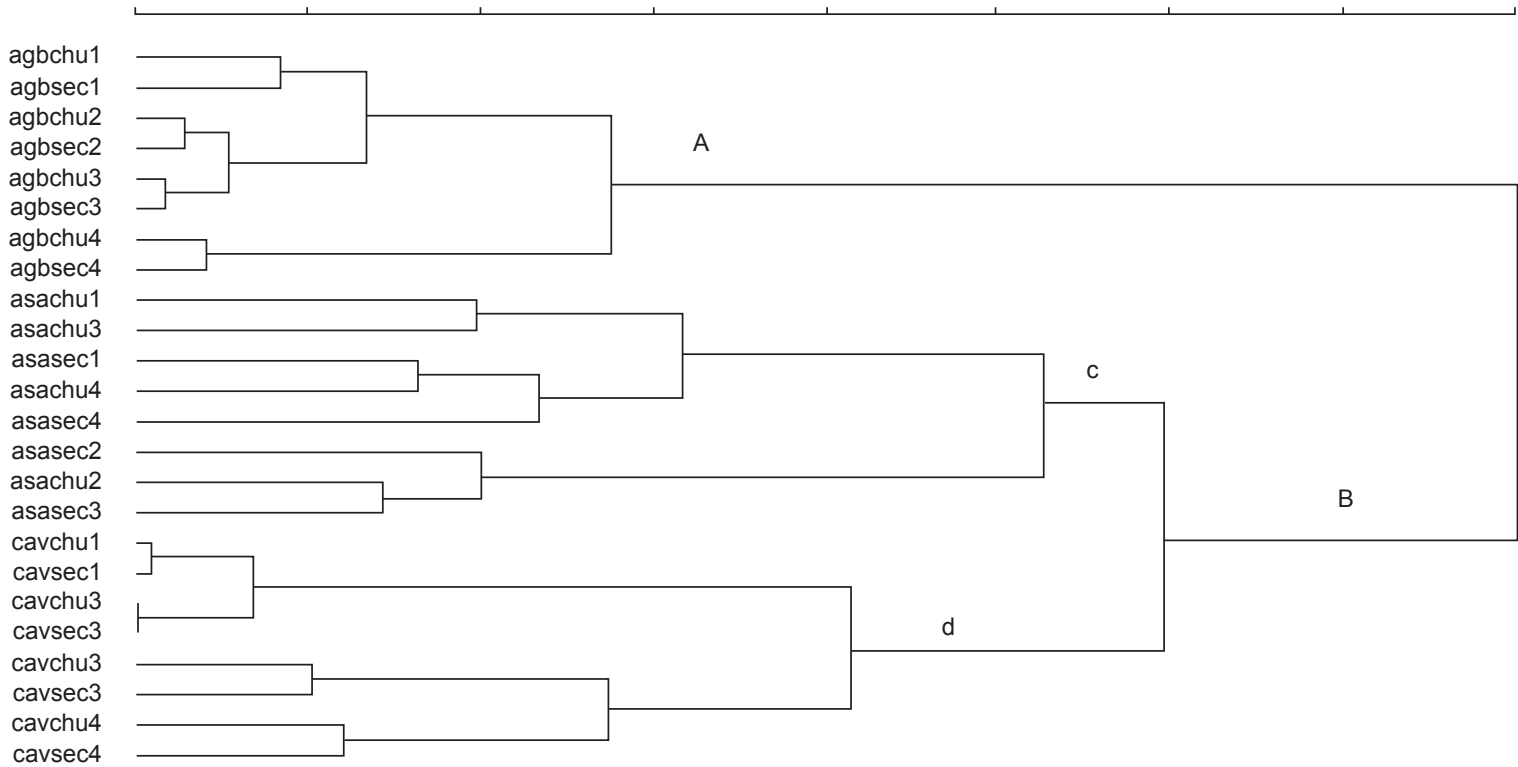

Figura 2. Dendrograma de similaridade baseado nas distâncias de Bray-Curtis (UPGMA) a partir das densidades populacionais temporais de cada riacho estudado. A explicação dos grupos formados (letras maiúsculas e minúsculas) encontra-se no texto.

Figure 2. Dendrogram of similarity inferred by Bray-Curtis distance (UPGMA) for temporal species density of each studied stream. Explanations of cluster groups (upper and lowercase letters) are presented along the text. 
apresentados temos exemplares testemunhos depositados na coleção ictiológica do Museu Nacional do Rio de Janeiro (MNRJ).

Considerando-se os três riachos estudados, foi registrado um total de 47 espécies, sendo que apenas 18 foram comuns aos três riachos. Os córregos Água Boa e Cavalo foram os que apresentaram mais espécies exclusivas, com seis registros. O córrego Acaba Saco apresentou apenas duas espécies exclusivas (Tabela 1). A análise de similaridade baseada na distância de Bray-Curtis para a composição de espécies de cada riacho foi tratada considerando-se os valores médios das densidades populacionais de cada riacho nos períodos de seca e chuva de cada um dos quatro anos de estudo. A partir dessa análise (Figura 2) obtivemos dois grandes grupos de localidades: i) grupo A que reuniu tanto os períodos de chuva como de seca dos quatro anos do córrego Água Boa e ii) grupo B que reuniu tanto os períodos de chuva como de seca dos quatro anos do córrego Acaba Saco e Cavalo. A observação desses resultados em um nível de similaridade menor indicou a subdivisão do grupo B em dois grupos menores (b e c) que distinguem os córregos Acaba Saco e Cavalo.

Tabela 1. Lista das espécies que ocorrem nos córregos Água Boa, Acaba Saco e Cavalo e respectivas densidades (ind.ha ${ }^{-1}$ ) e participação percentual (\%). As espécies destacadas em cinza claro e cinza escuro são espécies exclusivas do riacho em questão e comuns a todos os riachos, respectivamente.

Table 1. Species from streams Água Boa, Acaba Saco e Cavalo and their respectives densities (ind.ha ${ }^{-1}$ ) and percentual occurrence (\%). Grey and dark grey marked species are that exclusive at the mentioned stream and common to three studied streams, respectively.

\begin{tabular}{|c|c|c|c|c|c|c|c|c|}
\hline Água Boa & & & Acaba Saco & & & Cavalo & & \\
\hline & 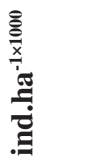 & $\%$ & & 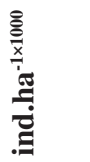 & $\%$ & & 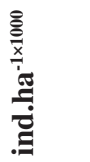 & $\%$ \\
\hline Ancistrus aguaboensis & 4,70 & 44,3 & Loricaria sp. & 2,02 & 19,6 & Ancistrus minutus & 4,3 & 36,5 \\
\hline Hypostomus plecostomus & 2,00 & 19,4 & Ancistrus minutus & 1,44 & 13,6 & Harttia punctata & 1,1 & 9,4 \\
\hline Harttia punctata & 0,54 & 5,1 & Harttia punctata & 1,12 & 10,9 & Hypostomus sp. 2 & 0,95 & 8,1 \\
\hline Loricaria sp. & 0,53 & 5,0 & Hypostomus sp.5 & 0,83 & 8,0 & Bryconamericus sp. & 0,78 & 6,7 \\
\hline Bryconamericus sp. & 0,48 & 4,6 & Hypostomus sp. 2 & 0,68 & 6,6 & Astyanax gr. bimaculatus & 0,70 & 6,0 \\
\hline Hypostomus sp. 2 & 0,29 & 2,7 & Cetopsorhamdia sp. & 0,65 & 6,3 & Imparfinis cf. schubarti & 0,60 & 5,1 \\
\hline Aspidoras sp. & 0,29 & 2,7 & Characidium sp.1 & 0,46 & 4,4 & Characidium sp.1 & 0,55 & 4,7 \\
\hline Knodus sp.1 & 0,26 & 2,5 & Hypostomus sp.3 & 0,41 & 3,9 & Knodus sp.1 & 0,48 & 4,0 \\
\hline Astyanax gr. bimaculatus & 0,24 & 2,2 & Corumbataia tocantinensis & 0,34 & 3,3 & Loricaria sp. & 0,44 & 3,7 \\
\hline Phenacorhamdia sp. & 0,22 & 2,0 & Astyanax gr. bimaculatus & 0,32 & 3,1 & Apareiodon machrisi & 0,38 & 3,2 \\
\hline Retroculus lapidifer & 0,18 & 1,6 & Phenacorhamdia sp. & 0,25 & 2,4 & Phenacorhamdia sp. & 0,24 & 2,0 \\
\hline Imparfinis cf. schubarti & 0,15 & 1,4 & Hypostomus plecostomus & 0,24 & 2,3 & Characidium sp.2 & 0,16 & 1,3 \\
\hline Creagrutus sp. & 0,09 & 0,8 & Mylesinus paucisquamatus & 0,24 & 2,3 & Acestrocephalus sp. & 0,14 & 1,1 \\
\hline Hypostomus sp.5 & 0,08 & 0,7 & Characidium sp.2 & 0,23 & 2,2 & Hypostomus sp.5 & 0,12 & 1,0 \\
\hline Apteronotus albifrons & 0,08 & 0,7 & Bryconamericus sp. & 0,20 & 1,9 & Knodus sp.5 & 0,11 & 0,9 \\
\hline Cichlasoma araguaiensis & 0,07 & 0,6 & Hypostomus sp.4 & 0,16 & 1,6 & Hypostomus plecostomus & 0,11 & 0,9 \\
\hline Mylesinus paucisquamatus & 0,07 & 0,6 & Retroculus lapidifer & 0,12 & 1,2 & Moenkhausia sp.1 & 0,09 & 0,7 \\
\hline Cetopsorhamdia molinae & 0,06 & 0,6 & Hoplias gr. malabaricus & 0,12 & 1,1 & Creagrutus sp. & 0,08 & 0,6 \\
\hline Moenkhausia sp.2 & 0,04 & 0,3 & Knodus sp.5 & 0,11 & 1,1 & Hoplias gr. malabaricus & 0,08 & 0,6 \\
\hline Knodus sp.5 & 0,02 & 0,2 & Cichlasoma araguaiensis & 0,09 & 0,9 & Hypostomus sp.3 & 0,07 & 0,5 \\
\hline Hypostomus sp.4 & 0,02 & 0,1 & Microlepidogaster sp. & 0,08 & 0,8 & Cetopsorhamdia sp. & 0,06 & 0,4 \\
\hline Hoplias gr. Malabaricus & 0,02 & 0,1 & Knodus sp.1 & 0,07 & 0,7 & Imparfinis borodini & 0,05 & 0,4 \\
\hline Pseudocetopsis sp. & 0,02 & 0,1 & Pseudocetopsis sp. & 0,05 & 0,4 & Moenkhausia sp.2 & 0,04 & 0,3 \\
\hline Gymnotus carapo & 0,01 & 0,1 & Apareiodon machrisi & 0,03 & 0,2 & Knodus sp.2 & 0,03 & 0,2 \\
\hline Moenkhausia sp.1 & 0,01 & 0,1 & Creagrutus sp. & 0,03 & 0,2 & Astyanax sp. & 0,01 & 0,1 \\
\hline Acestrocephalus sp. & 0,008 & 0,1 & Knodus sp.3 & 0,02 & 0,1 & Knodus sp.3 & 0,01 & 0,1 \\
\hline Cetopsorhamdia sp. & 0,006 & 0,1 & Astyanax sp. & 0,003 & 0,1 & Knodus sp.4 & 0,01 & 0,1 \\
\hline Characidium sp.1 & 0,006 & 0,1 & Gymnotus carapo & 0,003 & 0,1 & Rhamdia quelen & 0,008 & 0,1 \\
\hline Hypostomus sp.3 & 0,006 & 0,1 & & & & Pimelodella sp. & 0,006 & 0,1 \\
\hline Knodus sp.2 & 0,005 & 0,1 & & & & Cetopsorhamdia molinae & 0,004 & 0,1 \\
\hline Sternopygus macrurus & 0,005 & 0,1 & & & & Hypostomus sp.4 & 0,003 & 0,1 \\
\hline Apareiodon machrisi & 0,003 & 0,1 & & & & Leporinus ortomaculatus & 0,003 & 0,1 \\
\hline Hypostomus ericae & 0,003 & 0,1 & & & & Gymnotus carapo & 0,002 & 0,1 \\
\hline Rhamdia quelen & 0,003 & 0,1 & & & & Hemiodus ternetzi & 0,002 & 0,1 \\
\hline \multirow[t]{2}{*}{ Eigenmannia virescens } & 0,001 & 0,1 & & & & Leporellus vittatus & 0,002 & 0,1 \\
\hline & & & & & & Mylesinus paucisquamatus & 0,002 & 0,1 \\
\hline
\end{tabular}


As variações temporais das densidades das populações que compõem as comunidades apresentaram padrões distintos para cada riacho estudado. As análises de Spearman para os pares de meses de coleta revelaram que os córregos Água Boa e Cavalo apresentaram, respectivamente, 92 e $76 \%$ (166 e 138 de 180 pares de meses) dos casos significativamente correlacionados, indicando alta persistência temporal na estrutura das comunidades de peixes. Para o córrego Acaba Saco foi registrado 27\% (49 de 180 pares de meses) dos casos significativamente correlacionados, indicando baixa persistência temporal da estrutura da comunidade. Não foram registradas diferenças significativas entre as densidades médias de cada riacho estudado (Anova, $\mathrm{F}=0,24 ; \mathrm{p}=0,79$ ). Os valores médios das densidades totais de cada riacho variaram de 10.295 ind.ha ${ }^{-1}$ para o córrego Acaba Saco a 11.739 ind.ha ${ }^{-1}$ para o córrego Cavalo (Figura 3).

\section{Discussão}

Assumindo-se que os métodos amostrais fornecem dados representativos das comunidades, sabe-se que o número de espécies em rios/riachos é extremamente variável e depende, em parte, da região geográfica, do tipo de localidade amostrada e da posição da localidade de amostragem em relação às áreas de cabeceira (Matthews 1990). O número de espécies registrado no presente estudo está dentro da amplitude de valores registrada para outros riachos de regiões tropicais (e.g. Angermeier \& Karr 1983, Ibarra \& Stewart 1989, Wikramanayake 1990, Hugueny \& Paugy 1995, Castro \& Casatti 1997, Casatti et al. 2001, Fogaça et al. 2003, Barreto \& Aranha 2005, Langeani et al. 2007). Dentre as variações registradas na literatura, fica destacado que as menores diversidades ocorrem nas áreas de cabeceira ou próximas a ela enquanto nas áreas de desembocadura ou confluência o número de espécies é sempre superior (Matthews 1986, Edds 1993, Pavanelli \& Caramaschi 1997, Abes \& Agostinho 2001, Barreto \& Uieda 1998, Uieda \& Barreto 1999, Pavanelli \& Caramaschi 2003, Bifi et al. 2006, Perez-Jr. \& Garavello 2007). No caso do presente estudo, os três riachos estudados foram amostrados em áreas de baixada, próximo à confluência com o rio Tocantins, fato que pode explicar a alta diversidade como função da ictiofauna

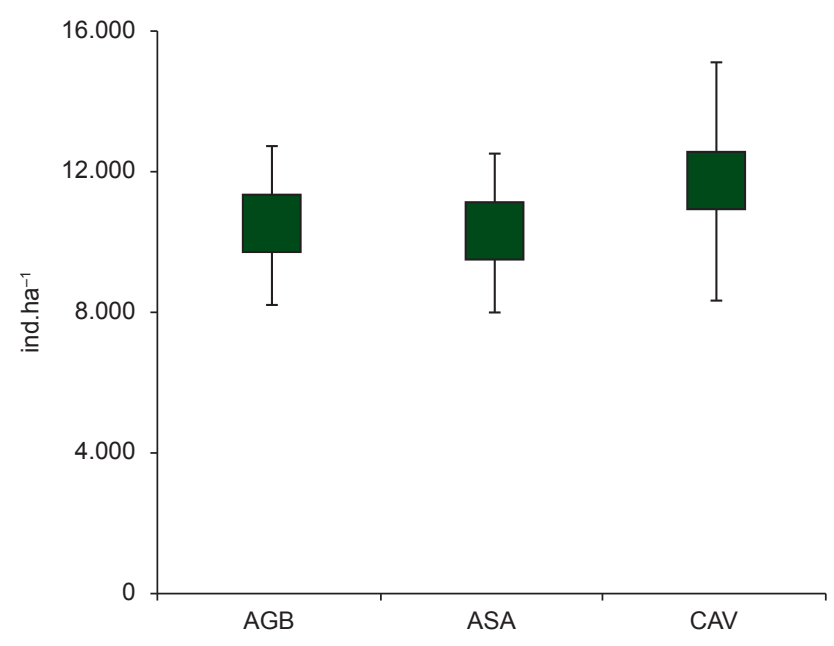

Figura 3. Valores médios das densidades das espécies que compõem as comunidades dos três riachos estudados no Alto RioTocantins. AGB = Água Boa, $\mathrm{ASA}=$ Acaba Saco e CAV = Cavalo.

Figure 3. Mean values of species densities from the three studied streams from the upper Tocantins River. AGB = Água Boa, ASA = Acaba Saco e $\mathrm{CAV}=$ Cavalo. deste rio de maior porte que sustenta grande diversidade de espécies ( 220 espécies - dados não publicados).

A análise comparativa das densidades médias de cada ano de estudo, para cada riacho estudado, não mostrou diferenças significativas, indicando alta persistência temporal na estrutura das comunidades de dois dos três riachos. Esse fato pode ser explicado por uma possível pré-adaptação das comunidades de peixes locais às oscilações hídricas sazonais. Fato como esse foi registrado em um sistema fluvial costeiro da bacia do Atlântico Leste, onde as espécies locais demonstraram estar bem adaptadas às enxurradas periódicas que tendem a promover alterações na estrutura física do hábitat (Mazzoni 1998, Mazzoni \& Lobón-Cerviá 2000). Enxurradas como as registradas nos riachos da bacia do Atlântico Leste não são comuns na região em que foi desenvolvido o presente estudo, no entanto, o regime de oscilação hídrica dessa área é marcado com estações seca e chuvosa claramente definidas que impõem constraints e respostas distintas às comunidades locais (Goulding 1980).

A análise das variações das densidades médias das principais espécies dos três riachos analisados indica o córrego Cavalo como aquele que sustenta maiores densidades de peixes (apesar da não significância das diferenças entre os três riachos). Entretanto, analisando-se comparativamente os dados de densidade média de populações de peixes de comunidades de diversas regiões biogeográficas, tratadas a partir da mesma metodologia usada no presente estudo (Tabela 2), obtém-se que os três riachos estudados, quando comparados com diversas localidades dos sistemas fluviais da região Tropical (Austral e Neotropical), estão entre os que suportam as menores densidades por área amostrada. Frente aos sistemas fluviais das regiões Holártica (Temperada e Mediterrânea) o padrão se repete o que coloca esses riachos como os de menores densidades dentre àqueles para os quais há dados disponíveis.

Randal et al. (1995) sugerem que as comunidades de peixes de rios são mais produtivas e suportam maiores densidades que as de lagos. No entanto, não existem dados conclusivos que permitam explicar essas diferenças (Mann \& Penczak 1986, Wellcomme 1992). Vários autores (Lowe McConnell 1987, Benke et al. 1988) sugerem maiores valores de biomassa (parâmetro correlacionado com a densidade) em ambientes tropicais como função das altas temperaturas e constância da radiação solar. Por outro lado, a comparação entre os dados da Tabela 2, com dados da bibliografia para produção de peixes em sistemas fluviais de regiões temperadas (Mahon \& Balon 1985, Mortensen 1977, Holcik 1996) indicam, com base na pouca informação disponível (i.e. Agostinho \& Penczack 1995, Mazzoni \& Lobón-Cerviá 2000, Schubarti \& Mazzoni 2006), que os ambientes tropicais apresentam os menores valores de densidade, sugerindo menores taxas de produtividade, quando comparados aos sistemas temperados (Mazzoni 1998).

Fatores como material orgânico bentônico (Watson \& Balon 1984) e fósforo (Randall et al. 1995) têm sido considerados os principais determinantes pelo aumento da produção em riachos. Provavelmente em função da estrutura geológica composta por formações précambrianas e corredeiras basálticas (Piccirillo et al. 1988) os riachos Neotropicais são pobres em nutrientes, livres de macrófitas e sujeitos a regimes pluviais marcados que agem, durante o período chuvoso, na "lavagem" do ambiente. Tem-se, ainda, que a disponibilidade de alimento autóctone é extremamente limitada em ambientes desse tipo, visto que a produção primária é facilmente carreada rio abaixo pela força do fluxo d'água durante a época de chuvas. Essas características são amplamente predominantes nos ambientes amazônicos (Goulding 1980), muito semelhantes aos riachos do presente trabalho, e podem explicar, em parte, o padrão de densidade apresentado para os córregos Acaba Saco, Cavalo e Água Boa. 
Miranda, J.C. \& Mazzoni, R.

Tabela 2. Riqueza de espécies ( $\mathrm{N}$ espécies) e densidades populacionais (ind.ha $\left.{ }^{-1} \times 1000\right)$ para riachos de diferentes regiões Neotropical, Austral e Holártica. Table 2. Species richness ( $\mathrm{N}$ espécies) and population densities (ind.ha $\left.{ }^{-1} \times 1000\right)$ for streams from the Neotropical, Austral and Holartic regions.

\begin{tabular}{|c|c|c|c|}
\hline Localidade & N espécies & ind.ha $\mathrm{a}^{-1(\times 1000)}$ & Referência \\
\hline \multicolumn{4}{|c|}{ Região neotropical - tropical (Bacia Amazônica) } \\
\hline Acaba Saco & 28 & 10,3 & Este estudo \\
\hline Cavalo & 36 & 11,7 & Este estudo \\
\hline \multirow[t]{2}{*}{ Água Boa } & 35 & 10,5 & Este estudo \\
\hline & média & 10,8 & \\
\hline \multicolumn{4}{|c|}{ Região neotropical - tropical (Bacia do Atlântico Leste) } \\
\hline Ubatiba 1 & 4 & 17,7 & Mazzoni \& Lobón-Cervià (2000) \\
\hline Ubatiba 2 & 14 & 27,9 & Mazzoni \& Lobón-Cervià (2000) \\
\hline Ubatiba 3 & 14 & 49,8 & Mazzoni \& Lobón-Cervià (2000) \\
\hline Ubatiba 4 & 13 & 15,1 & Mazzoni \& Lobón-Cervià (2000) \\
\hline Ubatiba 5 & 11 & 24,9 & Mazzoni \& Lobón-Cervià (2000) \\
\hline Ubatiba 6 & 14 & 23,9 & Mazzoni \& Lobón-Cervià (2000) \\
\hline Ubatiba 7 & 12 & 62,2 & Mazzoni \& Lobón-Cervià (2000) \\
\hline Ubatiba 8 & 16 & 57,1 & Mazzoni \& Lobón-Cervià (2000) \\
\hline \multirow[t]{2}{*}{ Ubatiba 9} & 15 & 70,3 & Mazzoni \& Lobón-Cervià (2000) \\
\hline & média & 38,8 & \\
\hline \multicolumn{4}{|c|}{ Região austral - tropical } \\
\hline Bornéo 1 & 32 & 20,6 & Watson \& Balon (1984) \\
\hline \multirow[t]{2}{*}{ Bornéo 2} & 28 & 27,0 & Bishop (1973) \\
\hline & média & 23,8 & \\
\hline \multicolumn{4}{|c|}{ Região holártica - mediterrânea } \\
\hline Argélia & 2 & 8,6 & Penczak \& Molinsky (1984) \\
\hline Bulgária 1 & 3 & 1,5 & Penczak et al. (1985) \\
\hline Bulgária 2 & 8 & 17,0 & Penczak et al. (1985) \\
\hline Espanha 1 & 3 & 20,9 & Rodriguez-Ruiz \& Granado (1991) \\
\hline Espanha 2 & 5 & 85,7 & Lobón-Cerviá \& Penczak (1984) \\
\hline Espanha 3 & 8 & 7,4 & Lobón-Cerviá et al. (1986) \\
\hline \multirow[t]{2}{*}{ Espanha 4} & 4 & 25,0 & Bravo (Dados não publicados) \\
\hline & média & 23,7 & \\
\hline \multicolumn{4}{|c|}{ Região holártica - temperada } \\
\hline Canadá & 15 & 273,3 & Mahon \& Balon (1985) \\
\hline Dinamarca & $3-7$ & 326,0 & Mortensen (1977) \\
\hline Slovakia & 6 & 542,0 & Hölcik (1996) \\
\hline \multirow[t]{2}{*}{ Polonia } & 20 & 117,6 & Penczak (1992) \\
\hline & média & 314,7 & \\
\hline
\end{tabular}

Com base nos resultados apresentados nesse estudo, concluímos que os riachos do alto Tocantins têm padrões de densidade distintos aos registrados para outros riachos da região Tropical estudados até o momento. No entanto, é evidente a necessidade de se ampliar o conhecimento sobre as comunidades de riachos dessa região além da importância de realizar análises mais específicas, relacionadas às taxas de produção secundária (=produção de peixes), para corroborar a proposta acima e incluir as comunidades de riachos tropicais em um contexto mais amplo da teoria ecológica.

\section{Agradecimentos}

Agradecemos a todos os membros dos Laboratórios de Ecologia de Peixes da UERJ e da UFRJ pelo auxílio no trabalho de campo e processamento de parte do material. Esse trabalho foi realizado através do contrato Serra da Mesa Energia-Furnas / BioRio / UFRJ, convênio UERJ, e é parte integrante da Dissertação do primeiro autor junto ao PPGB-Ecologia/UERJ.

\section{Referências Bibliográficas}

ABES, S.S. \& AGOSTINHO, A.A. 2001. Spatial patterns in fish distributions and structure of the ichthyocenosis in Água Nanci stream, upper Paraná river basin, Brazil. Hydrobiol. 445(3):217-227.

AGOSTINHO, A.A. \& PENCZAK, T. 1995. Populations and production of fish in two small tributaries of the Parana River, Paraná, Brazil. Hydrobiol. 312(3):153-166

ANGERMEIER, P.L. \& KARR, J.R. 1984. Fish communities along environmental gradients in a system of tropical streams. Env. Biol. Fish. 9(2):117-135.

APONE, F., OLIVEIRA, A.K. \& GARAVELLO, J.C. 2008. Ichthyofaunistic composition of the Quilombo river, tributary of the Mogi-Guaçu river, upper Paraná river basin, southeastern Brazil. Biot. Neotrop. 8(1):http:// 
www.biotaneotropica.org.br/v8n1/en/abstract?article+bn02208012008 (último acesso em 01/09/2009).

ARANHA, J.M.R. \& CARAMASCHI, E.P. 1997. Distribuição longitudinal e ocupação espacial de quatro espécies de Cyprinodontiformes no rio Ubatiba, Maricá, RJ. Act. Biol. Par. 26(1):125-140.

BARRETTO, M.G. \& UIEDA, V.S. 1998. Influence of the abiotic factors on the ichthyofauna composition in different orders stretches of Capivara River, São Paulo State, Brazil. Verh. Internat. Verein. Limnol. 26(1-5):2180-2183.

BARRETO, A.P. \& ARANHA, J.M.R. 2005. Assembléia de peixes de um riacho da Floresta Atlântica: composição e distribuição espacial (Guaraqueçaba, Paraná, Brasil). Acta Scient. Biol. Sci. 27(2):153-160.

BAYLEY, P.B. \& LI, H.W. 1992. Riverine fishes. In The river handbook: hydrological and ecological principles (P. Calow \& G.E. Petts, eds.). Blackwell Scientific Publications, New York, p. 251-281.

BIFI, A.G., BAUMGARTNER, D., BAUMGARTNER, G., FRANA, V.A. \& DEBONA, T. 2006. Composição específica e abundância da ictiofauna do rio dos Padres, bacia do rio Iguaçu, Brasil. Acta Scient. Biol. Sci. 28(3):203-211.

BISHOP, J.E. 1973. Limnology of a Malayan river Sungai Gombak. Kluwer Academic Publishers, London.

BENKE, A.C., HALL, C.A.S., HAWKINS, C.P., LOWE-McCONNELL, R.H., STANFORD, J.A., SUBERKROPP, K. \& WARD, J.V. 1988. Bioenergetic considerations in the analysis of stream ecosystems. Jour. North Am. Bent. Soc. 7(1-3):480-502.

BRAGA, F.M.S. \& ANDRADE, P.M. 2005. Distribuição de peixes na microbacia do ribeirão Grande, Serra da Mantiqueira Oriental, São Paulo, Brasil. Iheringia, Sér. Zool. 95(2):121-126.

CASATTI, L., LANGEANI, F. \& Castro, R.M.C. 2001. Peixes de riacho do Parque Estadual Morro do Diabo, bacia do Alto Rio Paraná, SP. Biot. Neotrop. 1(1): http://www.biotaneotropica.org.br/v1n12/pt/abstract?inv entory+BN-00201122001 (último acesso em 01/09/2009).

CASTRO, R.M.C. \& CASATTI, L. 1997. The fish fauna from a small forest stream of the upper Paraná River Basin, southeastern Brazil. Ichthyol. Explor. Freshw. 7(4):337-352.

EDDS, D.R. 1993. Fish assemblage structure and environmental correlates in Nepal's Gandaki River. Copeia. 1993(1):48-60.

FOGAÇA, F.N.O., ARANHA J.M.R. \& ESPER, M.L.P. 2003. Ictiofauna do Rio do Quebra (Antonina, PR, Brasil): ocupação espacial e hábito alimentar. Interciência. 28(3):168-173.GOULDING, M. 1980. The fishes and the forest: explorations in Amazonian Natural History. University of California Press, Berkeley.

HÖLCIK, J. 1996. Ecological fish production in the inland delta of the middle Danube, a floodplain system. Environm. Biol. Fish. 46(1):151-156.

HUGUENY, B. \& PAUGY, D. 1995. Unsaturated fish communities in African rivers. Am. Nat. 146(1):163-169.

IBARRA, M. \& STEWART, D.J. 1989. Longitudinal zonation of sandy beach fishes in the Napo River basin, eastern Ecuador. Copeia. 1989(2):364-381.

INNOCÊNCIO, N.R. 1977. Hidrografia. In Geografia do brasil: região centrooeste. IBGE, Rio de Janeiro, RJ.

LOBÓN-CERVIÀ, J. 1991. Dinamica de poblaciones de peces en rios: pesca eléctrica y métodos de capturas sucessivas en la estima de abundancias. Museo Nacional de Ciencias Naturales, Madrid, 156 p. (Monografia).

LOBÓN-CERVIÁ, J. \& PENCZAK, T. 1984. Fish production in the Jarama River, central Spain. Holarct. Ecology. 7(2):128-137.

LOBÓN-CERVIÁ, J., SOSTOA, A. \& MONTAÑÉS, C. 1986. Fish production and its relation with the community structure in a aquifer-fed stream of Old Castile (Spain). Pol. Arch. Hydrobiol. 33(3-4):333-343.

LOWE-McCONNELL, R.H. 1987. Ecological studies in tropical fish communities. Cambridge University Press, Cambridge.

MAHON, R. \& BALON, E.K. 1985. Fish production in warm-water streams in Poland and Ontario. Can. J. Fish. Aquat. Sci. 42(6):1211-1215.
MANN, R.H.K. \& PENCZAK, T. 1986. Fish production in rivers: a review. Pol. Arch. Hydrobiol. 33(2):233-247.

MATTHEWS, W.J. 1986. Fish faunal structure in an Ozark stream: stability, persistence and a catastrophic flood. Copeia. 1986(2):388-397.

MATTHEWS, W.J. 1990. Fish community structure and stability in a warmwater midwestern stream. In Ecology and assessment of warmwater streams: workshop synopsis. (M.B. Bain, ed.). Biol. Rep. 90(5):16-17.

MATTHEWS, W.J. 1998. Patterns in freshwater fish ecology. Chapman \& Hall, London.

MAZZONI, R. 1998. Estrutura das comunidades e produção de peixes de um sistema fluvial costeiro de Mata Atlântica, Rio de Janeiro. Tese de Doutorado, Universidade Federal de São Carlos, São Carlos.

MAZZONI, R. \& LOBÓN-CERVIÁ, J. 2000. Longitudinal structure, density and production rates of a neotropical stream fish assemblage: the river Ubatiba in the Serra do Mar, southeast Brazil. Ecography. 23(5):588-602.

MAZZONI, R., FENERICH-VERANI, N. \& CARAMASCHI, E.P. 2000. Electrofishing as a sampling technique for coastal stream fish populations and communities in the Southeast of Brazil. Rev. Brasil Biol. 60(2):205-216.

MAZZONI, R., FENERICH-VERANI, N., CARAMASCHI, E.P. \& IGLESIAS-RIOS, R. 2006. Stream-Dwelling fish communities from an Atlantic Rain Forest drainage. Brazil. Arch. Technol. Biol. 49(2):249-256

McCUNE, B. \& MEFFORD, M.J. 1997. PC-ORD: multivariate analysis of ecological data. Version 3,0. MjM Software Desogn, Gleneden Beach.

MIRANDA, J.C. \& MAZZONI, R. 2003. Composição da ictiofauna de três riachos do alto rio Tocantins - GO. Biota Neotrop. 3(1): http://www. biotaneotropica.org.br/v3n1/pt/abstract?article+BN00603012003 (último acesso em 01/09/2009).

MORTENSEN, E. 1977. Fish production in small Danish streams. Fol. Limnol. Scand. 17(1):21-26.

PAVANELLI, C.S. \& CARAMASCHI, E.P. 1997. Composition of the ichthyofauna of two small tributaries of the Paraná river, Porto Rico, Paraná state, Brazil. Ichthyol. Explor. Freshwaters 8(1):32-31.

PAVANELLI, C.S. \& CARAMASCHI, E.P. 2003. Temporal and spatial distribution of the ichthyofauna in two streams of the upper rio Paraná basin. Braz. Arch. Biol. Technol. 46(2):272-280.

PAYNE, A.L. 1986. The ecology of tropical lakes and rivers. John Wiley \& Sons, London.

PENCZAK, T. 1992. Fish production in the Warta River: postimpoundment study. Hydrobiol. 242(1):87-93.

PENCZAK, T., JANKOV, J. \& ZALEWSKI, M. 1985. Fish production in the Mesta River, Rila Mountain, Samokov, Bulgaria. Fish. Res. 3(2):201-221.

PENCZAK, T. \& MOLINSKY, M. 1984. Fish production in Oued Sebaou a seasonal river in North Algeria. J. Fish Biol. 25(3):723-732.

PEREZ Jr., O.R. \& GARAVELLO, J.C. 2007. Ictiofauna do ribeirão do Pântano, afluente do rio Mogi-Guaçu, bacia do alto rio Paraná, São Paulo, Brasil. Iheringia, Sér. Zool. 97(3):328-335.

PICCIRILLO, E.M., MELFI, A.J., COMIN-CHIARAMONTI, B., BELLIENI, G., ERNESTO, M., MARQUES, L.S., NARDIJ, J.R., PACCA, E.G., ROISENBERG, A. \& ESTOLFA, D. 1988. Continental flood volcanism from the Paraná basin. In Continental floods basalts (E.D. MacDougall, ed.). Kluwer Academic Publisher, Dordrecht, p. 195-238.

RANDAL, R.G., KELSO, J.R.M. \& MINNS, C.K. 1995. Fish production in freshwaters: Are rivers more productives than lakes? Can. J. Fish. Aquat. Sci. 52(3):631-643.

ROCHA, F.C., CASATTI, L. \& PEREIRA, D.C. 2009. Structure and feeding of a stream fish assemblage in Southeastern Brazil: evidence of low seasonal influences. Acta Limnol. Brasil. 21(1):123-134.

RODRIGUEZ-RUIZ, A. \& GRANADO, C. 1991. Fish production in a stream with Mediterranean regimen (SW Spain). Ecol. Inter. Bull. 19(1):65-78. 
SCHUBART, S.A. \& MAZZONI, R. 2006. Produtividade de peixes em um riacho costeiro da bacia do Leste, Rio de Janeiro, Brasil. Iheringia, Sér. Zool. 96(4):399-405.

SEBER, G.A.F. 1982. The estimation of animal abundance and related parameters. Griffin Co., London.

UIEDA, V.S. \& BARRETTO, M.G. 1999. Composição da ictiofauna de quatro trechos de diferentes ordens do rio capivara, bacia do Tietê, Botucatu, São Paulo. Rev. Bras. Zooc. 1(1):55-67.

VANNOTE, R.L., MINSHALL, G.W., CUMMINS, K.W., SENDEL, J.R. \& CUSHING, C.E. 1980. The river continuum concept. Can. J. Fish. Aquat. Sci. 37(3): 130-138.

WATSON, D.J. \& BALON, E.K. 1984. Structure and production of fish communities in tropical rain forest stream of north Borneo. Can. J. Zool. 62(5):927-940.
WELLCOMME, R. 1992. Pesca fluvial. FAO, Roma. (Documento Técnico de Pesca, n. 62.)

WIKRAMANAYAKE, E.D. 1990. Ecomorphology and biogeography of a tropical stream fish assemblage: evolution of assemblage structure. Ecology. 71(5):1756-1764.

ZAR, J.H. 1996. Biostatistical Analysis. 3 ed. Prentice Hall, London.

ZIPPIN, C. 1958. The removal method of population estimation. Journ. Wild. Manag. 22(1):82-90.

Recebido em 01/06/09 Versão reformulada recebida em 01/10/09

Publicado em 12/11/09 